\title{
Sodium Chlorite as an Efficient Oxidant and Hydroxy-ion Pump in Osmium-Catalyzed Asymmetric Dihydroxylation
}

\author{
Mikko H. Junttila and Osmo E. O. Hormi ${ }^{*}$
}

[*] Department of Chemistry

University of Oulu

P.O.Box 3000

FIN-90014 University of Oulu (Finland)

Fax: (+358) 085531603

E-mail: osmo.hormi@oulu.fi 
Table of Contents:

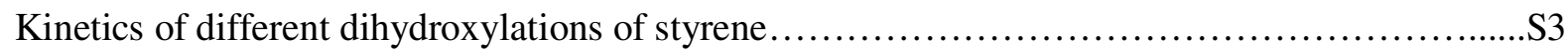

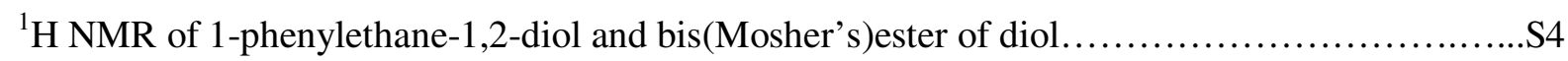

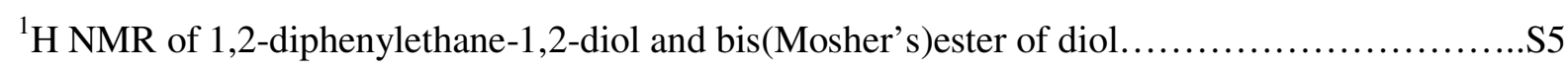

${ }^{1} \mathrm{H}$ NMR of 2-phenylpropane-1,2-diol and (Mosher's)ester of diol................................. 6

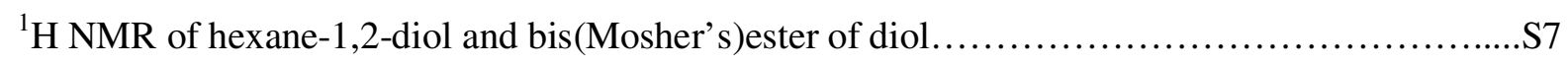

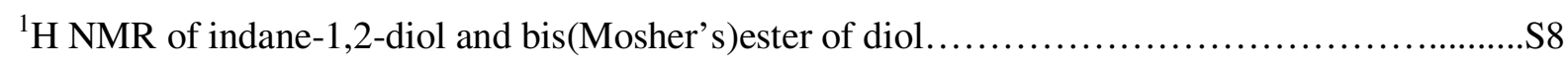

${ }^{1}$ H NMR of 1-methyl cyclohexane-1,2-diol and (Mosher's)ester of diol..........................S9

${ }^{1}$ H NMR of hexadecan-1,2-diol and bis(Mosher's)ester of diol.................................. 10

${ }^{1} \mathrm{H}$ NMR of methyl 2,3-dihydroxy-3-phenylpropanoate and bis(Mosher's)ester of diol.............S11 


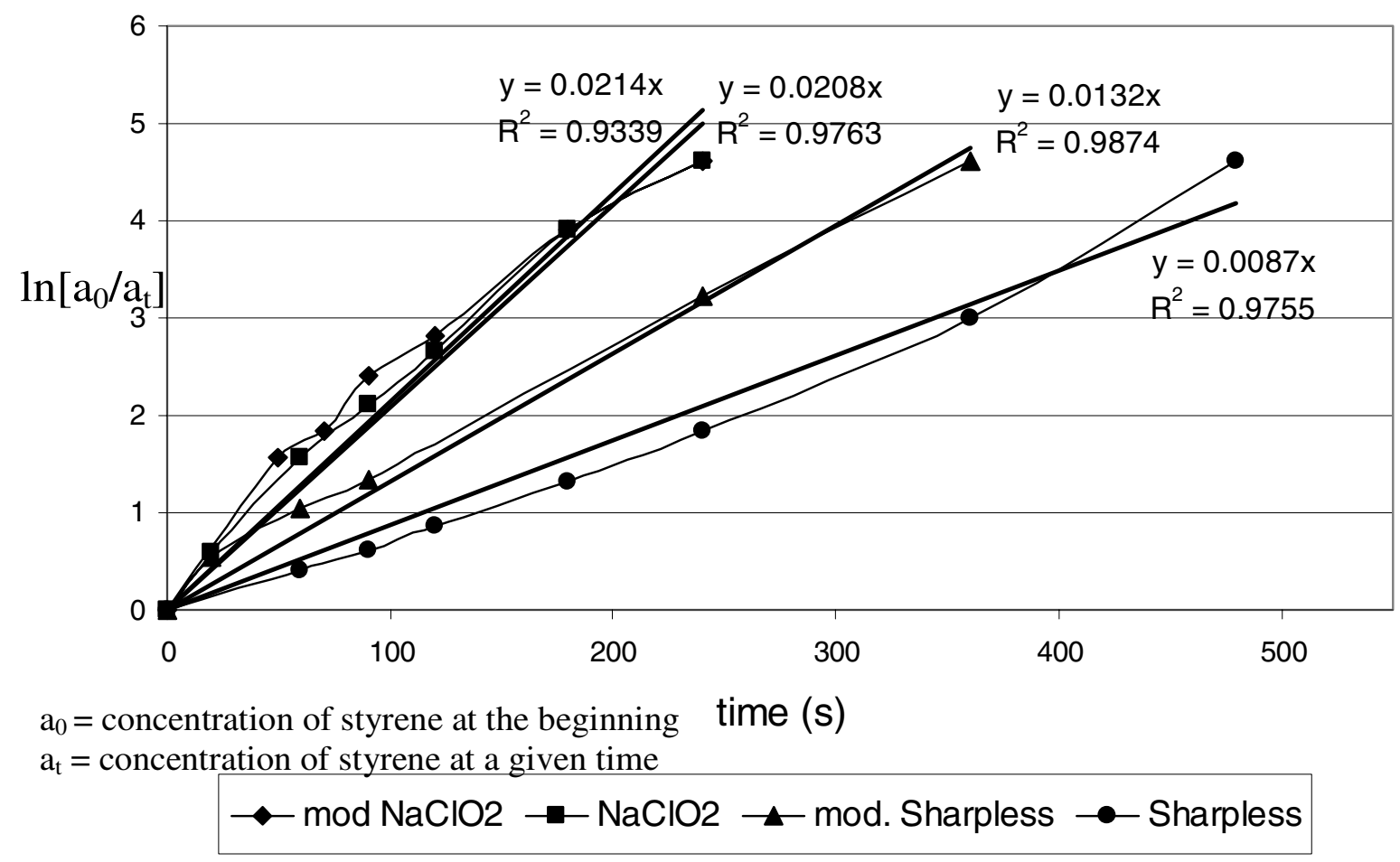

Figure S-1. Kinetics of different dihydroxylations of styrene. 
1-phenylethane-1,2-diol: HRMS (M+Na) 161.0570, calculated 161.0578
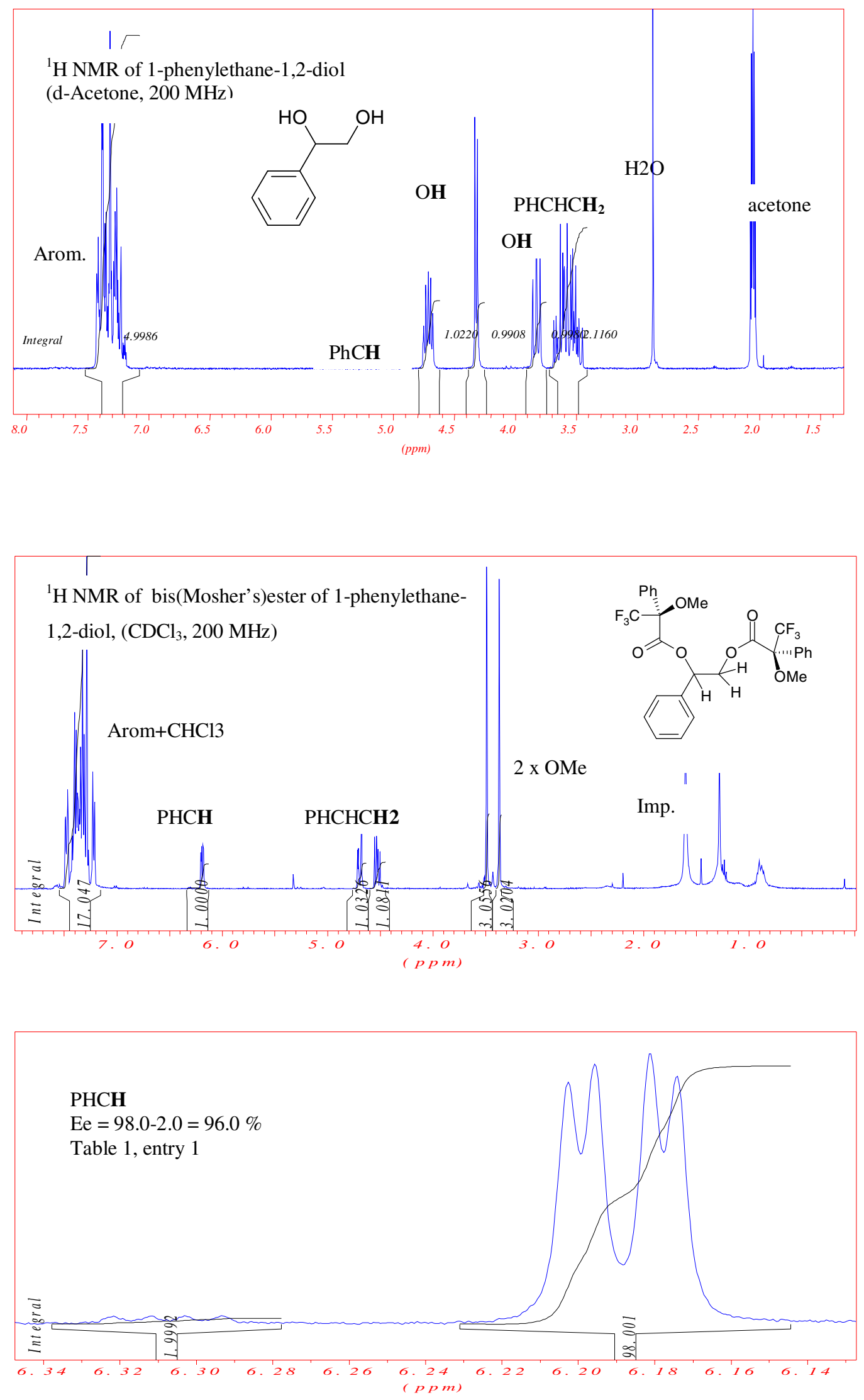
1,2-diphenylethane-1,2-diol: HRMS (M+Na) 237.0869, calculated $237.0891\left(\mathrm{C}_{14} \mathrm{H}_{14} \mathrm{O}_{2} \mathrm{Na}\right)$
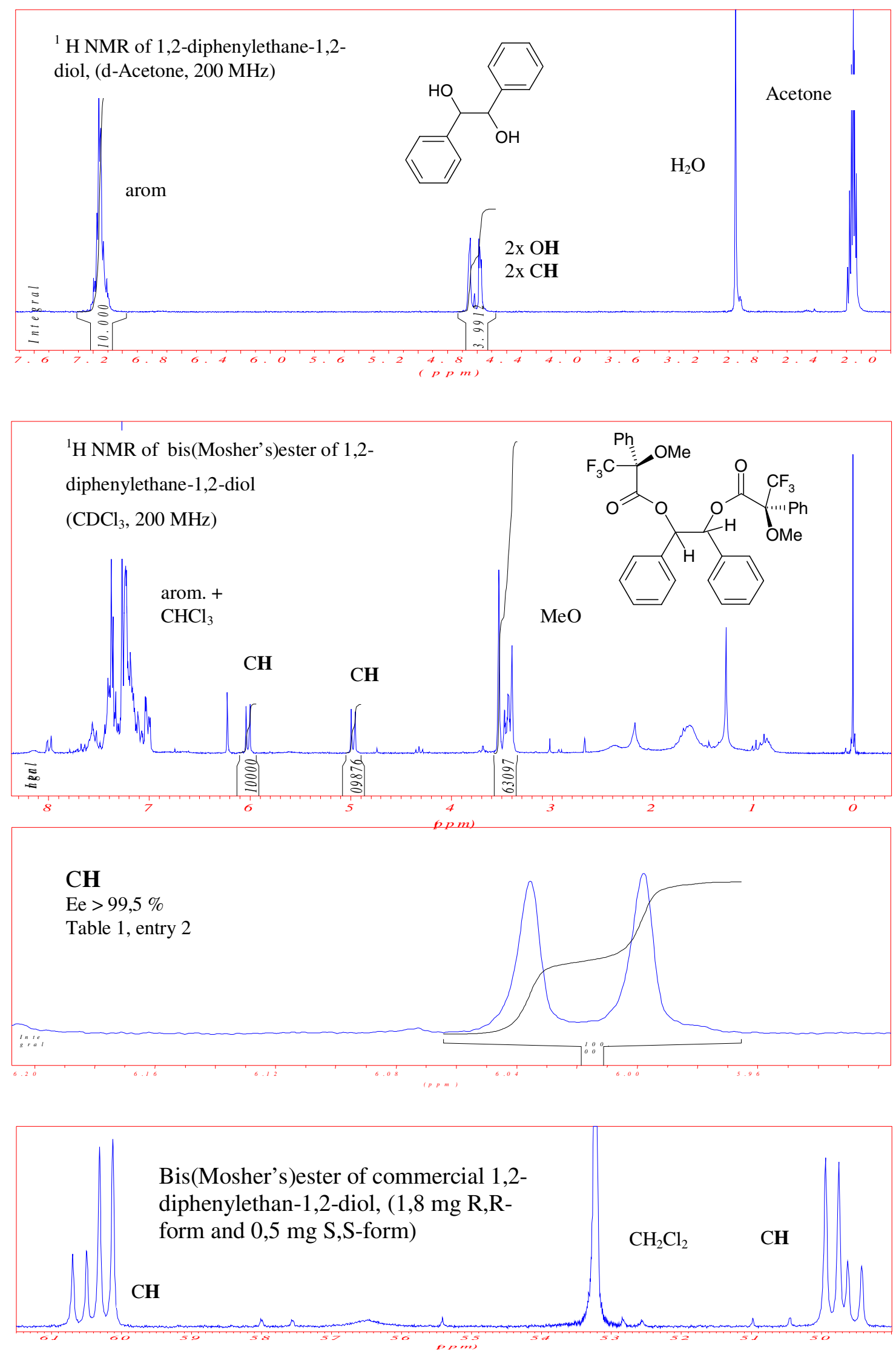
2-phenylpropane-1,2-diol: HRMS (M+Na) 175.0728, calculated $175.0735\left(\mathrm{C}_{9} \mathrm{H}_{12} \mathrm{O}_{2} \mathrm{Na}\right)$
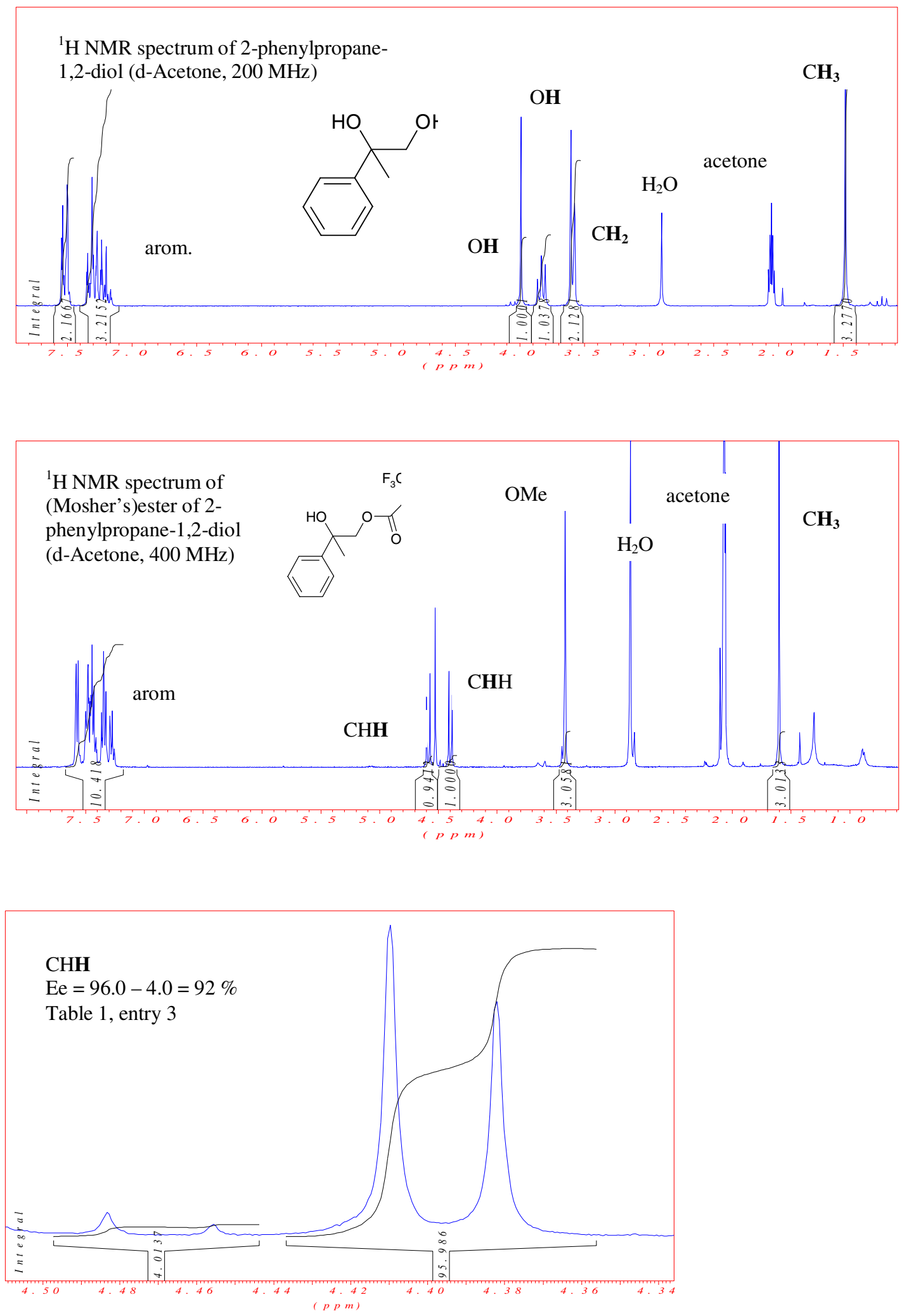
Hexane-1,2-diol: HRMS (M+Na) 141.0903, calculated $141.0891\left(\mathrm{C}_{6} \mathrm{H}_{14} \mathrm{O}_{2} \mathrm{Na}\right)$
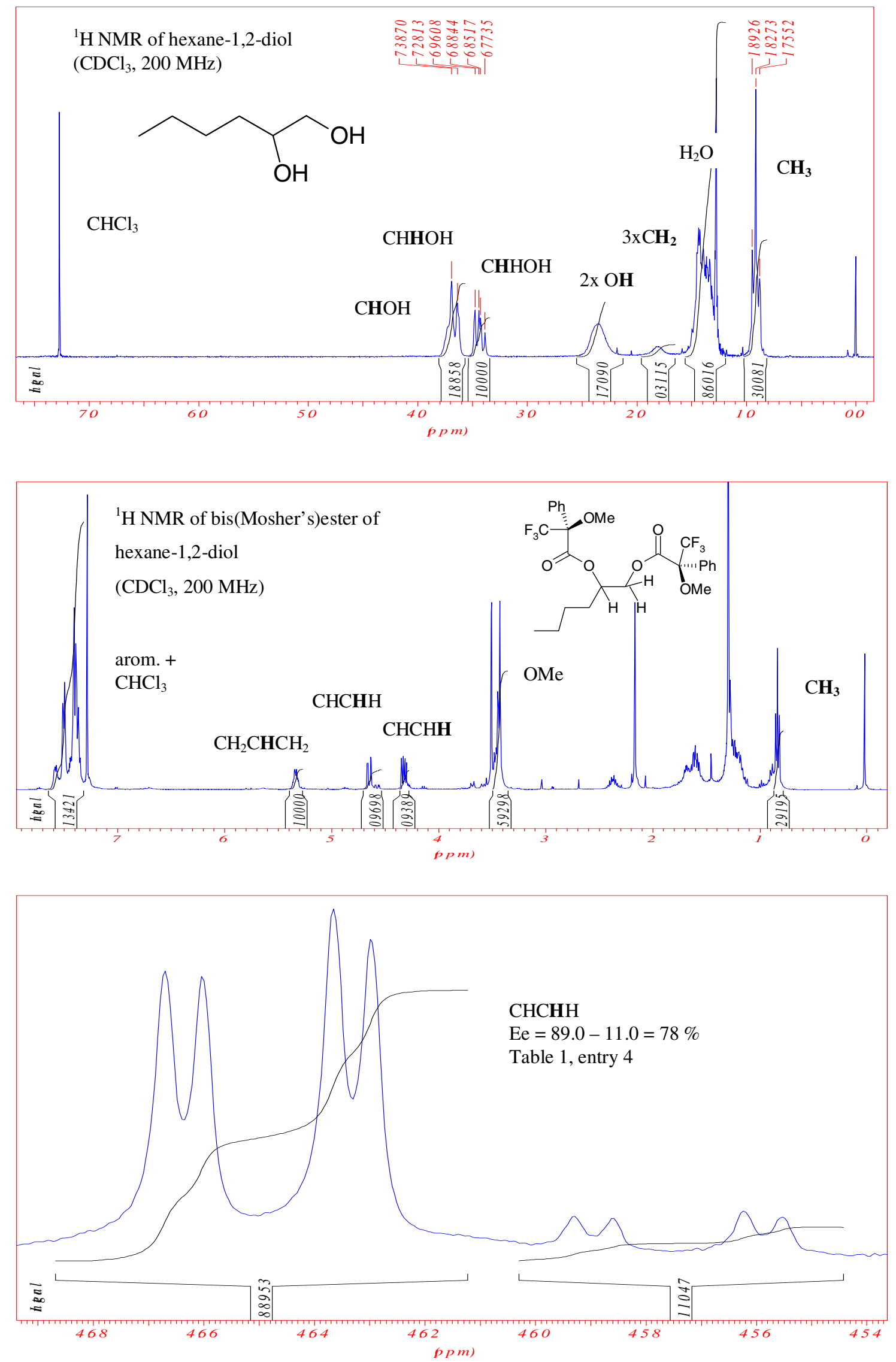
Indane-1,2-diol: HRMS (M+Na) 173.0597, calculated $173.0578\left(\mathrm{C}_{9} \mathrm{H}_{10} \mathrm{O}_{2} \mathrm{Na}\right)$
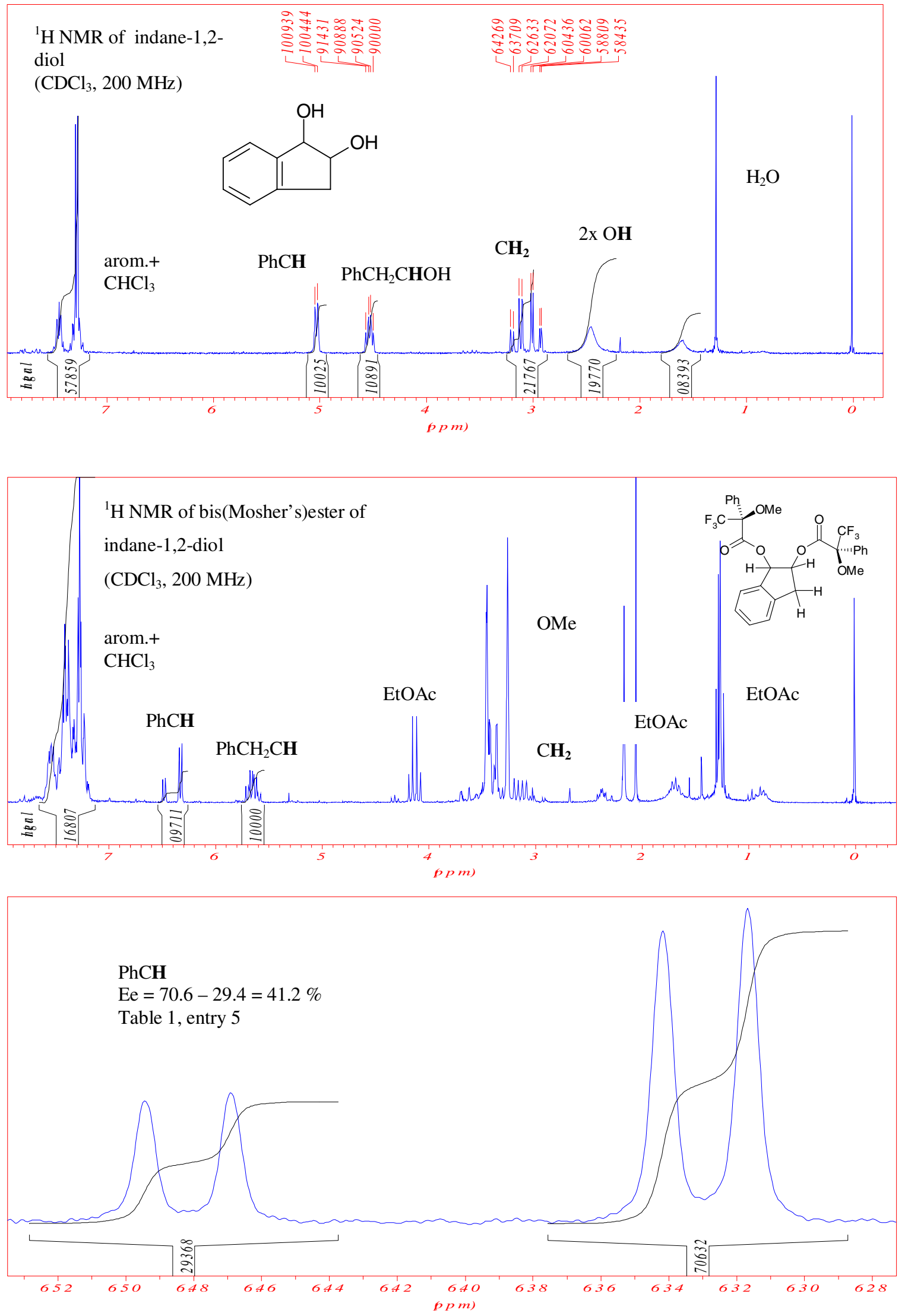
1-methyl cyclohexane-1,2-diol: HRMS (M+Na) 153.0918, calculated $153.0891\left(\mathrm{C}_{7} \mathrm{H}_{14} \mathrm{O}_{2} \mathrm{Na}\right)$
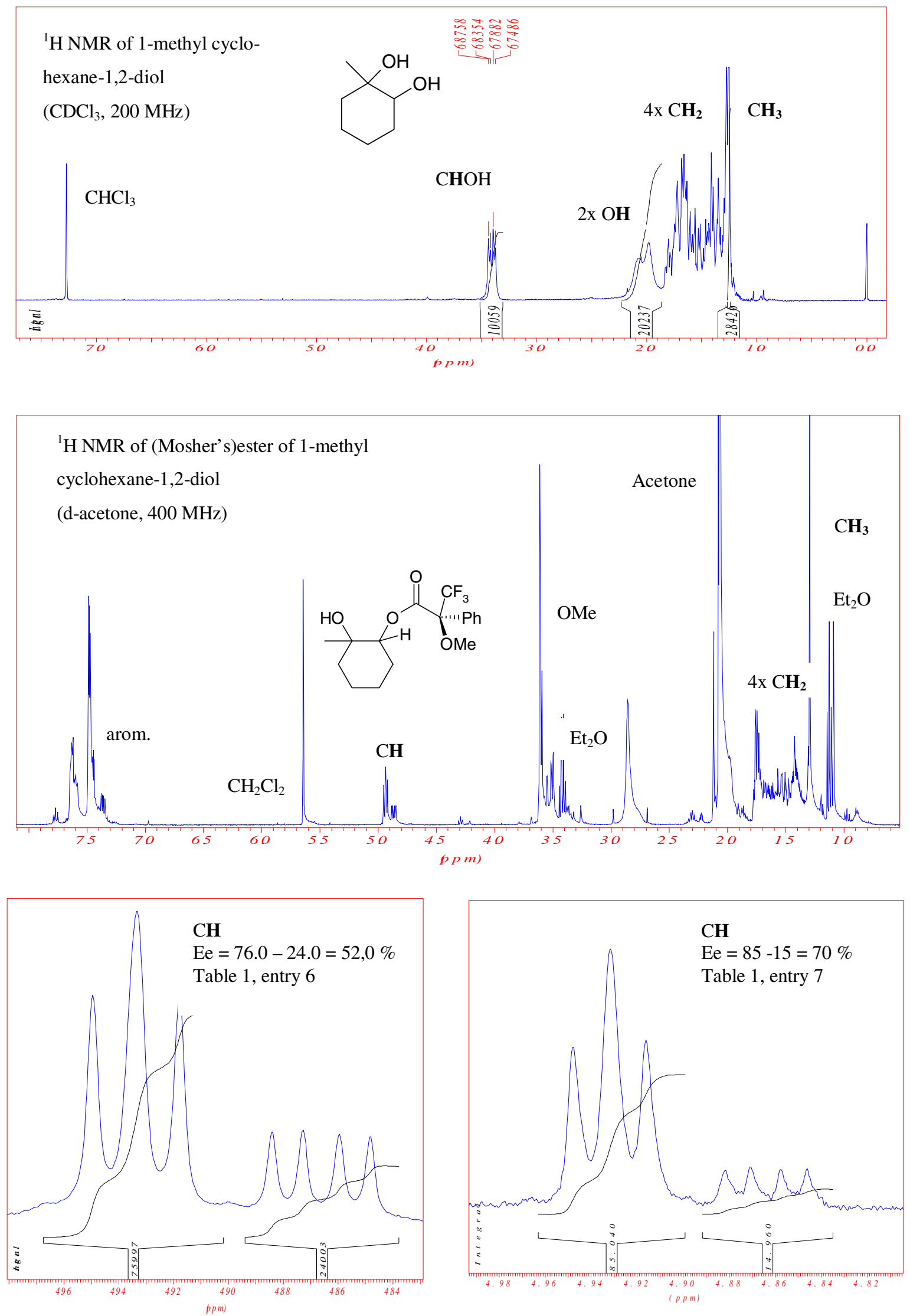
Hexadecan-1,2-diol: HRMS (M+Na) 281.2463, calculated 281.2457 $\left(\mathrm{C}_{16} \mathrm{H}_{34} \mathrm{O}_{2} \mathrm{Na}\right)$
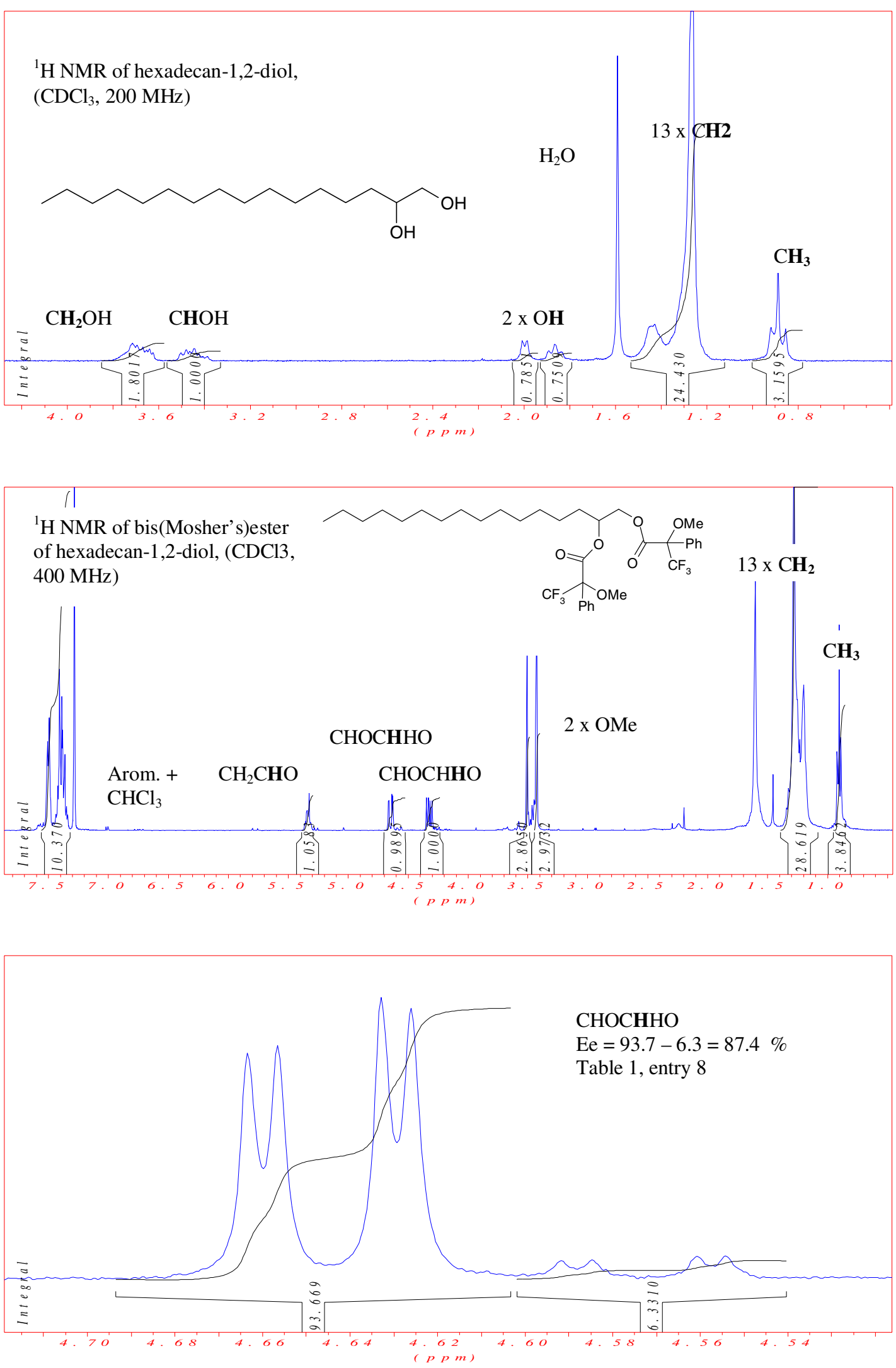
Methyl 2,3-dihydroxy-3-phenylpropanoate: HRMS (M+Na) 219.0628, calculated 219.0633 $\left(\mathrm{C}_{10} \mathrm{H}_{12} \mathrm{O}_{4} \mathrm{Na}\right)$
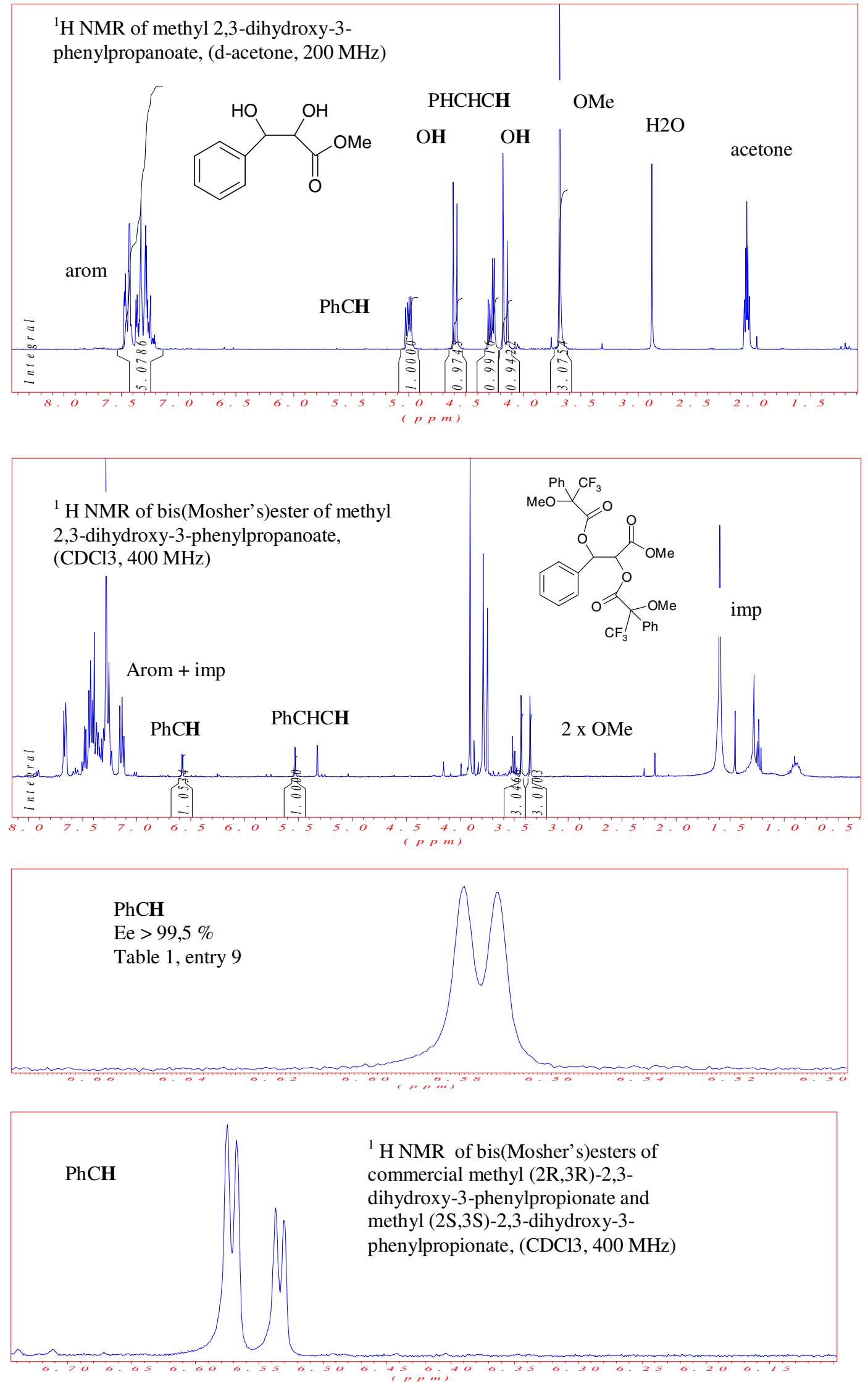